\title{
A mathematical model of magnetohydrodynamic micropolar fluid motion via permeable media with Soret and Dufour effects
}

\author{
Ram Prakash Sharma ${ }^{1 *}$, S.R. Mishra ${ }^{2}$ \\ ${ }^{1}$ Department of Mathematics, JECRC University Jaipur, India \\ ${ }^{2}$ Department Mathematics, Siksha 'O' Anusandhan Deemed to be University, Khandagiri, Bhubaneswar 751030, Odisha, India
}

Corresponding Author Email: ramprakash0808@gmail.com

https://doi.org/10.18280/mmc b.870406

Received: 1 June 2017

Accepted: 5 January 2018

\section{Keywords:}

porous media, micropolar fluid, MHD, soret and dufour effect, heat source/sink

\begin{abstract}
In this article, we have examined 2-dimensional steady magnetohydrodynamic boundary layer motion of viscous micropolar liquid through an extending surface. Simultaneous impacts of Soret and diffusion-thermo are considered. Furthermore, the impact of heat source/sink and first order chemical reaction are also examined. The basic numerical problem i.e. structure of PDE's is transformed nonlinear into ODE's through using appropriate transformations. The changed governing equations are explained mathematically through $\mathrm{R}-\mathrm{K}$ fourth order method. The effect of different parameters on momentum, microrotation, energy, concentration descriptions, shear stress, transfer rate of heat and mass are examined through graphs. Mathematical evaluation is furthermore examined through the existing available outcome as a particular case of our research work.
\end{abstract}

\section{INTRODUCTION}

The thermal buoyancy caused because of heating/cooling of a perpendicular surface has a huge influence on motion and energy transport properties. Convective heat transport in the liquid motion is a trend of considerable attention from simultaneously hypothetical and realistic point of perspective due to its enormous demands in countless manufacturing and geophysical areas. Mixed free and forced convective past a plane surface has been broadly investigated from equally theoretical and tentative point of view through the over few years. The impact of a magnetic field on natural convective transfer of heat on isothermal perpendicular surface has been examined by Sparrow and Cess [1]. Boundary layer natural convective motion of an electrically conducting liquid over a perpendicular sheet under the influence of identical exterior heat flux and changeable wall heat was investigated by Gupta [2]. Char [3], Chiam [4], Liu [5], Ishak et al. [6] and Prasad et al. [7] have analyzed transfer of energy and flow properties past an extending surface with a consistent magnetic field. The overhead analysis examined the motion entirely generated by a stretching surface saturated in a different state latent motion. Assorted convective laminar motion on a vertical sheet via permeable media in the presence of slip situation has been analyzed by Harris et al. [8]. The laminar slip motion past a flat surface in the occurrence of stable temperature flux was studied by Aziz [9]. Most recently Rohni et al. [10] analyzed numerically the unsteady assorted convective laminar motion close to 2-D stagnation end on a perpendicular porous sheet immersed in a liquid-muddy permeable medium by temperature slip effect and suction. Outstanding reviews of the combined convective MHD motion in vertical surface have been studied by various researchers [11-15]. Ishak et al. [16] investigated MHD motion of micropolar liquid to stagnation end lying on a vertical sheet. Steady assorted convective laminar motion of a micropolar liquid close to the stagnation end on a perpendicular sheet was investigated by Lok et al. [17]. Ramachandran et al. [18] analyzed assorted convective in a stagnation motion close to perpendicular sheet. Each and every one of the over current examinations are associated to micropolar liquid over a perpendicular sheet. The MHD motion in a vertical curved permeable area with temperaturedependent heat source under the influence of slip-flow boundary condition has been examined by Srinivas and Muthuraj [19]. They have also analyzed the impact of MHD assorted convection peristaltic motion in a vertical permeable space under the influence of chemical reaction [20]. Combined transfer of energy and mass over a free convective in a permeable media has stimulated significant observation in the past many years, because of its huge prime engineering and geophysical practical utilities. Nield and Bejan [21], Ingham and Pop [22-23] and Bejan and Khair [24] have studied an inclusive assessment on this area. However, in past few decades researchers worked on Dufour and Soret impact those cannot be abandoned [Eckert and Drake [25]. Considering the significance of aforesaid influences, Kafoussias and Williams [26] investigated on energy and mass transport motion in occurrence of heat reliant viscosity. Anghel et al. [27] have analyzed the thermo diffusion and diffusion thermo influences on natural convective laminar flow over a vertical surface with saturated porous media. Postelnicu [28-29] have examined the result of MHD and chemical reaction on an electrically conducting viscous liquid past a vertical surface embedded in a permeable media. Alam and Rahman [30] have analyzed the thermo diffusion and Dufour impact on assorted convective motion over a perpendicular permeable flat sheet under the influence of variable suction. The dual scattering on free convection of energy and mass transport from a perpendicular surface flooded by non-Darcy electrically conducting liquid fixed porous media under the impact of thermo diffusion and Dufour influence has been examined by Murthy et al. [31] The impact of thermo diffusion and Dufour on laminar 
magnetohydrodynamic convection motion through a rotating disk was examined by Rashidi et al. [32]. The impacts of energy and mass transport on 2-D unsteady MHD natural convection motion over a perpendicular permeable surface in a porous media with diffusion- thermo effects was studied by Mishra et al. [33].

The originality of the current work is to bring out the influence of porous medium with Soret and Dufour influences on the energy and mss transport characteristic of natural convective micropolar liquid over a perpendicular sheet. The outcome of Ishak et al. [6], Ramchandran et al. [18] and Baag et al. [34] examined as individual cases of the current work. An additional objective of the current work is the technique of explanation. The R-K technique followed by shooting method is providing the similar result as that of the solution obtained by Ishak et al. [6] which was solved by finite difference technique.

\section{FORMULATION OF THE PROBLEM}

The steady, 2-D, viscous incompressible electrically conducting micropolar liquid close to a stagnation end on a perpendicular hot sheet is studied (Fig.1). 2- Identical and conflicting forces are functional through $x$ - axis in order that the surface is extending retaining the source permanent. The magnetic Reynolds number of the conducting fluid is supposed chosen very little in order that Hall effect and induced magnetic field may be abandoned. Therefore, magnetic field effect in momentum is considered in the current learning. Furthermore, the motion field is conditional on identical oblique magnetic field $B_{0}$ is concerned normal to the sheet. Examinations of magnetohydrodynamics boundary layer motion throughout smooth sheet and in the flooded area of bodies under the influence of transverse magnetic field reveal that the magnetic field decreases skin friction and energy transport and enhance the sock detachments span.

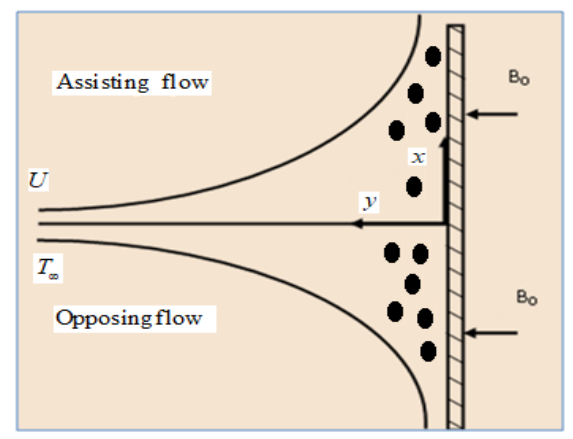

Figure 1. Flow configuration

Following Bhattacharyya [35] the governing equations of flow, heat and mass for free convective in Boussinesq's approximation with boundary conditions are

$\frac{\partial u}{\partial x}+\frac{\partial v}{\partial y}=0$

$u \frac{\partial u}{\partial x}+v \frac{\partial u}{\partial y}=U \frac{d U}{d x}+\left(\frac{\mu+\kappa}{\rho}\right) \frac{\partial^{2} u}{\partial y^{2}}+\frac{\kappa}{\rho} \frac{\partial N}{\partial y}+\left(\frac{\sigma B_{0}^{2}}{\rho}+\frac{v}{K_{p}^{*}}\right)(U-u) \pm g \beta_{T}\left(T-T_{\infty}\right) \pm g \beta_{c}\left(C-C_{\infty}\right)$

$\rho j\left(u \frac{\partial N}{\partial x}+v \frac{\partial N}{\partial y}\right)=\gamma^{\prime} \frac{\partial^{2} N}{\partial y^{2}}-\kappa\left(2 N+\frac{\partial u}{\partial y}\right)$ $u \frac{\partial T}{\partial x}+v \frac{\partial T}{\partial y}=\alpha \frac{\partial^{2} T}{\partial y^{2}}+\frac{D K_{T}}{C_{S} C_{p}} \frac{\partial^{2} C}{\partial y^{2}}+S^{\prime}\left(T-T_{\infty}\right)$

$u \frac{\partial C}{\partial x}+v \frac{\partial C}{\partial y}=D \frac{\partial^{2} C}{\partial y^{2}}+\frac{D K_{T}}{T_{m}} \frac{\partial^{2} T}{\partial y^{2}}-K_{c}^{*}\left(C-C_{\infty}\right)$

$u=0, v=0, N=-\frac{1}{2} \frac{\partial u}{\partial y}, T=T_{w}(x), C=C_{w}(x)$,

$$
\left.\begin{array}{l}
\text { at } y=0 \\
\text { as } y \rightarrow \infty
\end{array}\right\}
$$$$
u \rightarrow U(x), N \rightarrow 0, T \rightarrow T_{\infty}, C \rightarrow C_{\infty}
$$

The flow function $\psi(x, y)$ is introduced as

$u=\frac{\partial \psi}{\partial y}, v=-\frac{\partial \psi}{\partial x}$

Now, we introduce the following dimensionless variables

$$
\begin{aligned}
& \xi=\left(\frac{a}{v}\right)^{1 / 2} y, f(\xi)=\frac{\psi}{(a v)^{1 / 2} x}, \omega(\xi)=\frac{N}{a(a / v)^{1 / 2} x}, \theta(\xi)=\frac{T-T_{\infty}}{T_{\omega}-T_{\infty}}, \varphi(\xi)=\frac{C-C_{\infty}}{C_{\omega}-C_{\infty}}, M=\frac{\sigma B_{0}^{2}}{\rho a}, \gamma=\frac{K_{c}^{*}}{a}, \\
& \lambda=\frac{G r}{\operatorname{Re}_{x}^{2}}, G r=\frac{g \beta_{T}\left(T_{\omega}-T_{\infty}\right) x^{3}}{v^{2}}, G c=\frac{g \beta c\left(C_{\omega}-C_{x}\right) x^{3}}{v^{2}}, \delta=\frac{G c}{\operatorname{Re}_{x}^{2}}, l=\frac{v}{a}, \operatorname{Re}_{x}=\frac{U x}{v}, S=\frac{S}{a}, S c=\frac{v}{D} \\
& K_{p}=\frac{v}{a K_{p}^{*}}
\end{aligned}
$$

For relation in (7), the continuity Eq. (1) is satisfied identically and (2)-(6) yield

$$
\begin{aligned}
& (1+\Gamma) f^{\prime \prime \prime}+f f^{\prime \prime}+1-f^{\prime 2}+\Gamma \omega^{\prime}+\left(M+K_{p}\right)\left(1-f^{\prime}\right) \pm \lambda \theta \pm \delta \varphi=0 \\
& \left(1+\frac{\Gamma}{2}\right) \omega^{\prime \prime}+f \omega^{\prime}-f^{\prime} \omega-\Gamma\left(2 \omega+f^{\prime \prime}\right)=0 \\
& \frac{1}{\operatorname{Pr}} \theta^{\prime \prime}+f \theta^{\prime}-f^{\prime} \theta+S \theta+D u \varphi^{\prime \prime}=0
\end{aligned}
$$

$\frac{1}{S c} \varphi^{\prime \prime}+f \varphi^{\prime}-f^{\prime} \varphi-\gamma \varphi+S r \theta^{\prime \prime}=0$

where

$$
D u=\frac{D K_{T}\left(C_{\omega}-C_{\infty}\right)}{\operatorname{Cs} C p v\left(T_{\omega}-T_{\infty}\right)}, S r=\frac{D K_{T}\left(T_{\omega}-T_{\infty}\right)}{T_{m} v\left(C_{\omega}-C_{\infty}\right)} \quad \Gamma=\frac{\kappa}{\mu},
$$

Substituting Eq. (8) into Eq. (6), the boundary situations are as follows:

$$
\left.\begin{array}{lll}
f(0)=0, f^{\prime}(0)=0, \omega(0)=-\frac{1}{2} f^{\prime \prime}(0), \theta(0)=1, \varphi(0)=1, & \text { at } & \eta=0 \\
f^{\prime}(\eta) \rightarrow 1, \omega(\eta) \rightarrow 0, \theta(\eta) \rightarrow 0, \varphi(\eta) \rightarrow 0 & \text { as } & \eta \rightarrow \infty
\end{array}\right\}
$$

where prime stand for diff. w. r. to $\eta$.

\subsection{Material quantities of importance}

The major material quantities of attention are explained as $C_{f}=\frac{\tau_{w}}{\frac{\rho U_{w}^{2}}{2}}\left(\right.$ Skin friction coefficient $\left.C_{f}\right)$,

$N u=\frac{x q_{w}}{k\left(T_{w}-T_{\infty}\right)}($ Nusselt number $N u)$,

$S h=\frac{x q_{m}}{D_{m}\left(C_{w}-C_{\infty}\right)}($ Sherwood number $S h)$ 
where outside shear stress, outside heat and mass flux are explained as

$$
\tau_{w}=\left[(\mu+\kappa) \frac{\partial u}{\partial y}+\kappa N\right]_{y=0}, q_{w}=-k\left(\frac{\partial T}{\partial y}\right)_{y=0}, q_{m}=-D_{m}\left(\frac{\partial C}{\partial y}\right)_{y=0}
$$

Employing the non-dimensional variables (8), we obtain from Eqs. (14) and (15) as

$$
\frac{1}{2} C_{f} \operatorname{Re}_{x}^{1 / 2}=\left(1+\frac{\Gamma}{2}\right) f^{\prime \prime}(0), \frac{N u}{\operatorname{Re}_{x}^{1 / 2}}=-\theta^{\prime}(0), \frac{S h}{\operatorname{Re}_{x}^{1 / 2}}=-\varphi^{\prime}(0),
$$

\section{RESULTS AND DISCUSSION}

Two dimensional MHD flow of micropolar fluid under the influence of porous matrix and identical heat source has been studied. The inclusion of Dufour and thermo-diffusion effects makes the governing equations coupled, highly nonlinear and complex. The exact solution of these equations does not hold good. Therefore, numerical technique is implemented to get approximate solution. Eqs. (9) - (12) with the help of Eq. (13) are changed to set of first order differential equations and next explained mathematically using $4^{\text {th }}$-order R-K technique. In this section the numerical computations of the emerging variables are obtained and presented through graphs. Further, the shear and couple stresses, for both the momentum profiles, rate of energy and mass transport are in addition dispensed through Figures. Stagnation point flow of free convective steady micropolar fluid subject to oblique magnetic field with identical heat basis was studied. The objective of the paper reveals the influence of variables presented such as Soret $(\mathrm{Sr})$, Dufour $(D u)$ variables also the additional parameters come into view in the free convection diffusion problem. Moreover, the case of Newtonian fluid $(\Gamma=0)$ can also be derived as a particular case of Baag et al. [34] (Table-1). Fig.2 dispenses the influence of material parameter on velocity profiles for both Newtonian $(\Gamma=0)$ and non-Newtonian $(\Gamma \neq 0)$ fluid in the nonappearance of optimism variables and both the occurrence / nonappearance of permeable medium. It is obvious to observe that the velocity description decreases with an increase in $\Gamma$. For $\Gamma=0$, i.e. in case of Newtonian fluid velocity contributes its maximum value near the plate. In the present case the Soret and Dufour effects are withdrawn, as well as in the absence of porous matrix $(\mathrm{Kp}=0)$ the current outcome is differentiate with the previous available outcome of Baag et al. [34]. Though, under the influence of permeable matrix, the velocity boundary film thickness also decreases. Fig. 3 accords the microrotation profiles in reply to nonNewtonian parameter again in the absence of Soret, Dufour number, buoyancy parameters and both absence/attendance of permeable matrix. It is observed that the microrotation profile has a reverse trend as that of velocity distribution upto a region $\eta=1.25$ where it is interesting to remark that point of inflection occurs and afterwards its behavior is similar to that of velocity profile. Porosity is a resistive force which withstands the liquid motion close to the plate. Figs. 4 and 5 exhibit the variation of velocity and microrotation profiles for the material parameter in assisting case $(\lambda, \delta \geq 1)$ i.e. the presence of buoyancy variables and under the influence of permeable medium. It is clear to observe that non-Newtonian parameter has a decelerating effect as describe in Fig.2 for velocity profile. The increase in the heat and concentration buoyancy parameter, the boundary layer thickness decreases significantly under the influence of permeable matrix (Fig.4). From Fig.5 it is to note that in assisting case i.e. increase in buoyancy parameters the microrotation profile decreases within the region $\eta=1.25$ and thereafter effect is reversed. In the second region the effect is not significant and it meets the requisite boundary conditions. Fig. 6 displays the impact of material parameter and the heat and mass buoyancy parameters on the temperature description of micropolar fluid under the influence of porous matrix and absence of heat source/sink. It is noted from the figure that the fluid temperature has an enhancing effect with a raise in substance variable. Moreover, enhance in buoyant forces the thermal boundary layer thickness reduces in the presence of permeable matrix. The contribution of material parameter and the buoyant forces on the mass profile is very alike to that of energy description and displayed in Fig.7. Fig. 8 displays the temperature profile for the variation of heat source/sink under the influence/absence of permeable matrix. It is obvious to mark that enhance in source, heat of the micropolar liquid increases significantly whereas the impact is opposite in case of sink i.e. sink retards the fluid energy in the presence/nonattendance of permeable matrix. But presence of permeable matrix has a slight decelerating effect on the temperature profiles. The role of Soret and diffusion-thermo on the profiles are vital and interesting in the current study. The influence of diffusion-thermo on the temperature profile is reveals in Fig.9 under the influence of sink. It clear to remarked that because of enhancement in Dufour number the fluid temperature enhances significantly. The description is asymptotic in nature to assemble the boundary conditions. Fig.10 exhibits the impact of thermo-diffusion on the concentration description. Due to enhance in Soret number the temperature of the fluid increases in assisting flow. Thus, it is concluded that the inclusion of Dufour and Soret numbers are beneficial for the enhancement in fluid temperature and concentration. Fig. 11 describes the compound reaction effect on the mass profiles. It is observed that destructive chemical reaction $(\gamma>0)$ has a retarding impact on the concentration profile and incase of constructive $(\gamma<0)$ the profile enhances significantly. One prominent outcome is that the positive reaction merges with opposing buoyancy force produce a higher rate of increase in the concentration profile. Thus, constructive chemical reaction inclusion with opposing force is favorable to enhance the concentration description at every points in its boundary film. Figs. 12-14 present the variation of different variables on the skin friction, couple stress, rate of energy and concentration transfer. Fig. 12 presents the effect of thermal buoyancy parameter with variation of magnetic parameter. It is noticed that the skin friction coefficient, couple stress and rate of energy transfer increases by the enhance during thermal buoyancy parameter as magnetic parameter increases whereas rate of mass transfer decreases significantly. In this regard thermo-diffusion plays a vital role which contributes a fall in rate of mass transfer. Fig. 13 illustrates the variation of both the buoyant parameters with the variation of Soret number. It is observed is similar to that of Fig. 12 but one striking feature is that the rate of mass transfer has a dual character with different values of Soret number within the range 0 to 1 . From $S r=0$ to 0.75 rate of concentration transport enhances by the enhancing value of buoyant parameters whereas after that the profile decreases. This clear observation is remarked from Fig.14. It is noticed that the variation of concentration buoyancy parameter with the 
enhance in thermal buoyancy within the range 0 to 1 . Thus, it is concluded that the interaction of buoyant parameters, Soret and Dufour number enhances the properties of micropolar fluid which is clear in this study.

Table 1. Comparison of $f^{\prime \prime}(0)$ for various parameters

\begin{tabular}{c|c|c|c|c|c|c|c|c|c}
\hline $\operatorname{Pr}$ & $\lambda$ & $\delta$ & $S c$ & $S$ & $\Gamma$ & $\gamma$ & Present & $\begin{array}{c}\text { Baag et } \\
\text { al. }\end{array}$ & $\begin{array}{c}\text { Ramchandran } \\
\text { et al. }\end{array}$ \\
\hline 0.71 & 0 & 0 & 0.22 & 0 & 0 & 0 & 1.70328 & 1.70328 & 1.70637 \\
7 & 0 & 0 & 0.22 & 0 & 0 & 0 & 1.518 & 1.5179 & 1.517952 \\
\hline
\end{tabular}

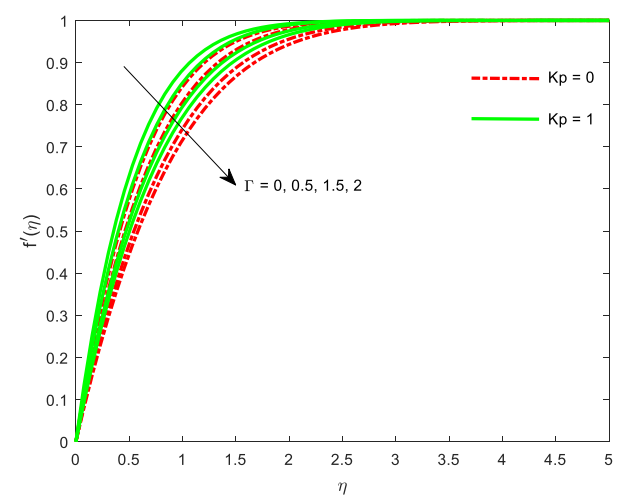

Figure 2. Influence of $\Gamma$ and $\mathrm{Kp}$ on velocity profiles for $\mathrm{M}=1 ; \operatorname{Pr}=1, \lambda=0 ; \delta=0 ; \mathrm{S}=0 ; \mathrm{Sc}=0.22 ; \gamma=0 ; \mathrm{Sr}=0 ; \mathrm{Du}=0$

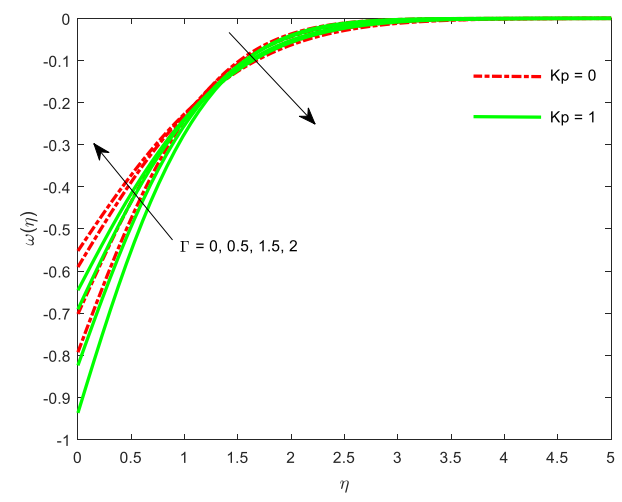

Figure 3. Influence of $\Gamma$ and $\mathrm{Kp}$ on microrotation description for $\mathrm{M}=1 ; \mathrm{Pr}=1, \delta=0 ; \lambda=0 ; \mathrm{S}=0 ; \mathrm{Sc}=0.22 ; \gamma=0 ; \mathrm{Sr}=0 ; \mathrm{Du}=0$

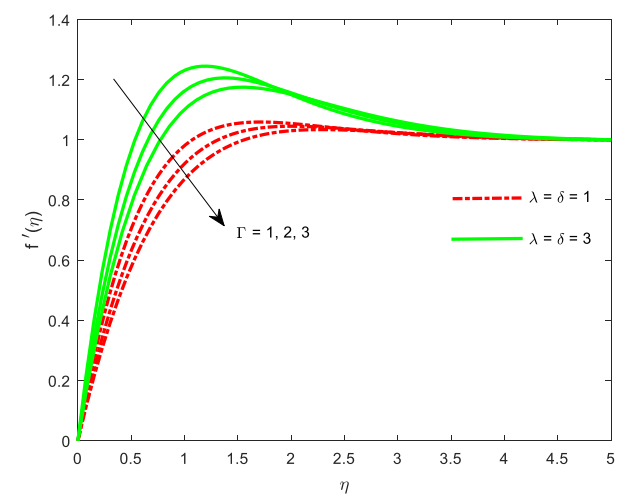

Figure 4. Influence of $\Gamma$ and $\mathrm{Kp}$ on velocity descriptions for $\mathrm{M}=1 ; \mathrm{Pr}=1 ; \mathrm{S}=0 ; \mathrm{Sc}=0.22 ; \gamma=0 ; \mathrm{Sr}=0 ; \mathrm{Du}=0$

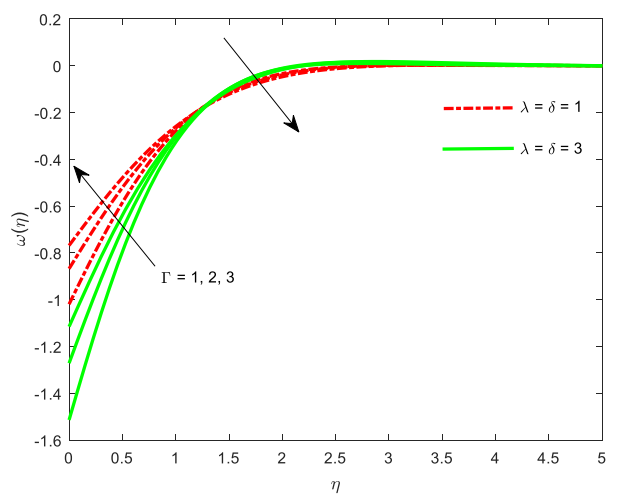

Figure 5. Influence of $\Gamma$ and $\mathrm{Kp}$ on microrotation profiles for $\mathrm{M}=1 ; \mathrm{Pr}=1 ; \mathrm{S}=0 ; \mathrm{Sc}=0.22 ; \gamma=0 ; \mathrm{Sr}=0 ; \mathrm{Du}=0$

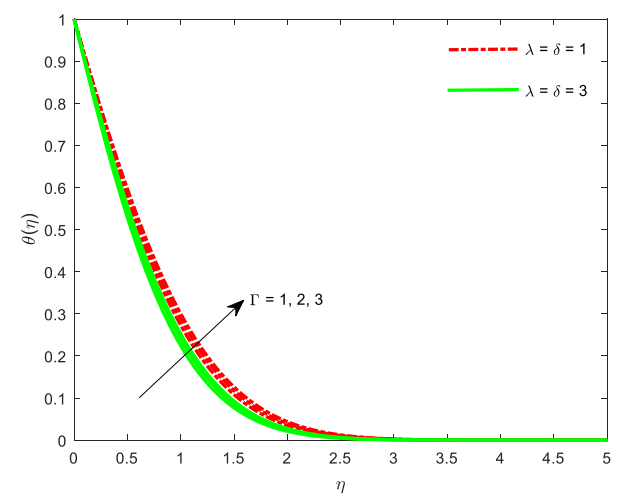

Figure 6. Influence of $\Gamma$ and $\mathrm{Kp}$ on temperature profiles for $\mathrm{M}=1 ; \mathrm{Pr}=1 ; \mathrm{S}=0 ; \mathrm{Sc}=0.22 ; \gamma=0 ; \mathrm{Sr}=0 ; \mathrm{Du}=0$

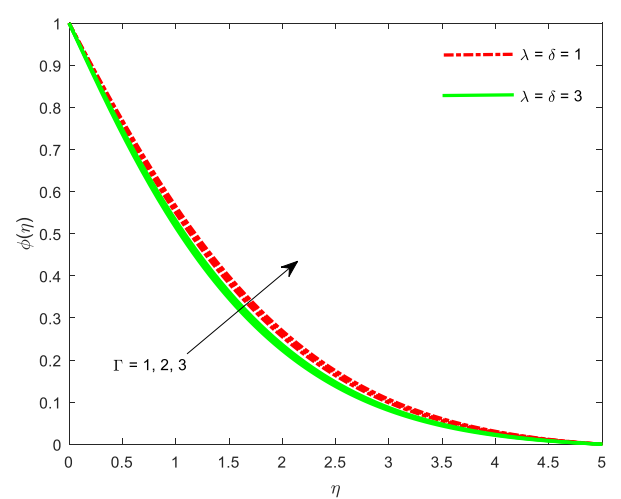

Figure 7. Influence of $\Gamma$ and $\mathrm{Kp}$ on concentration description for $\mathrm{M}=1 ; \mathrm{Pr}=1 ; \mathrm{S}=0 ; \mathrm{Sc}=0.22 ; \gamma=0 ; \mathrm{Sr}=0 ; \mathrm{Du}=0$

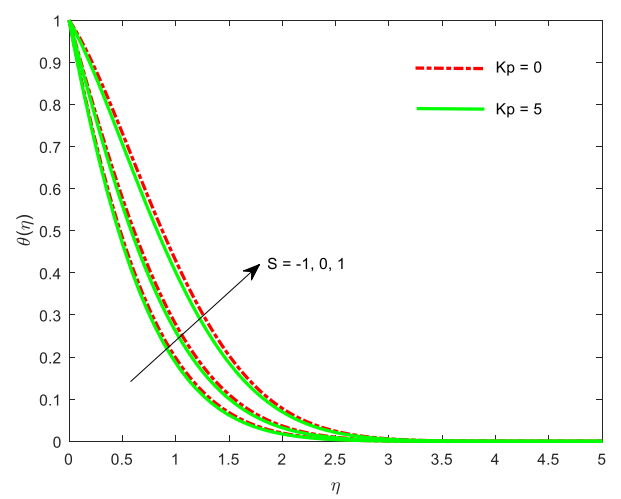

Figure 8. Influence of $\mathrm{S}$ and $\mathrm{Kp}$ on temperature description for $\mathrm{M}=1 ; \operatorname{Pr}=1, \delta=1 ; \lambda=1 ; \mathrm{S}=0 ; \mathrm{Sc}=0.22 ; \gamma=0 ; \mathrm{Sr}=0 ; \mathrm{Du}=0$ 


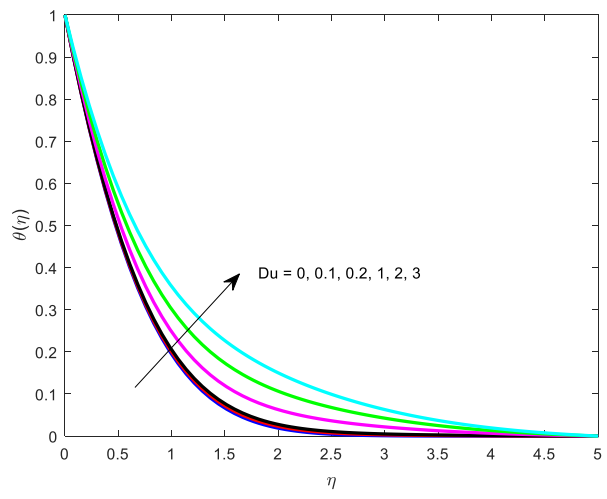

Figure 9. Influence of $\mathrm{Du}$ on-temperature profiles for $\mathrm{M}=1$; $\mathrm{Pr}=1, \delta=1 ; \lambda=1 ; \mathrm{S}=-1 ; \mathrm{Sc}=0.22 ; \gamma=0 ; \mathrm{Sr}=0 ; \mathrm{Du}=0$

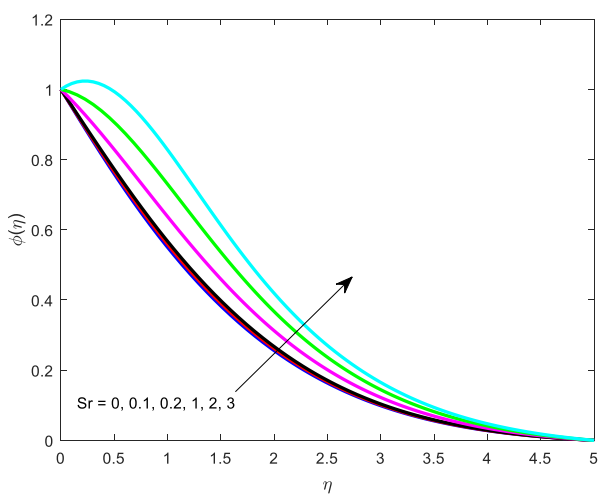

Figure 10. Influence of $\mathrm{Sr}$ on concentration descriptions for $\mathrm{M}=1 ; \operatorname{Pr}=1, \delta=1 ; \lambda=1 ; \mathrm{S}=-1 ; \mathrm{Sc}=0.22 ; \gamma=0 ; \mathrm{Du}=0.5$

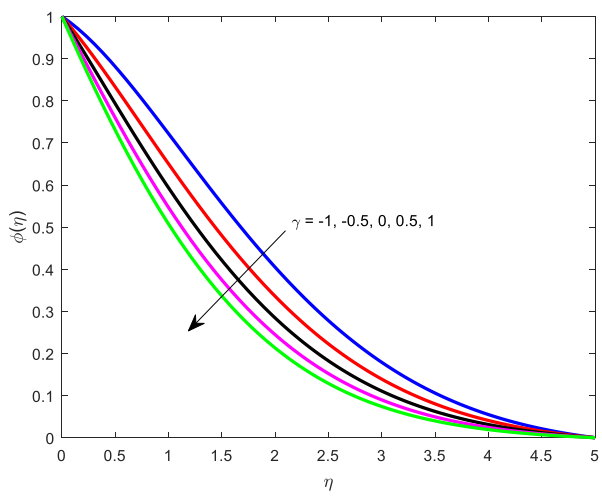

Figure 11. Influence of $\gamma$ on concentration descriptions for $\mathrm{M}=1 ; \mathrm{Kp}=1 ; \mathrm{Pr}=1, \lambda=\delta=1 ; \mathrm{Sc}=0.22 ; \mathrm{Sr}=0.5 ; \mathrm{Du}=0.5 ; \mathrm{S}=$ 1
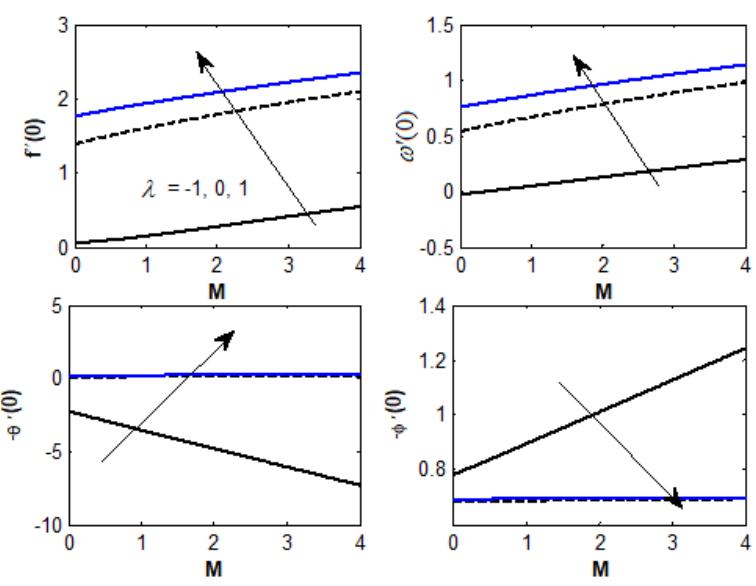

Figure 12. Influence of $\mathrm{M}$ on profiles for $\mathrm{Kp}=1 ; \operatorname{Pr}=1, \mathrm{D}=1$; $\mathrm{Sc}=0.22 ; \mathrm{Sr}=0.5 ; \mathrm{Du}=0.5 ; \mathrm{S}=-1$
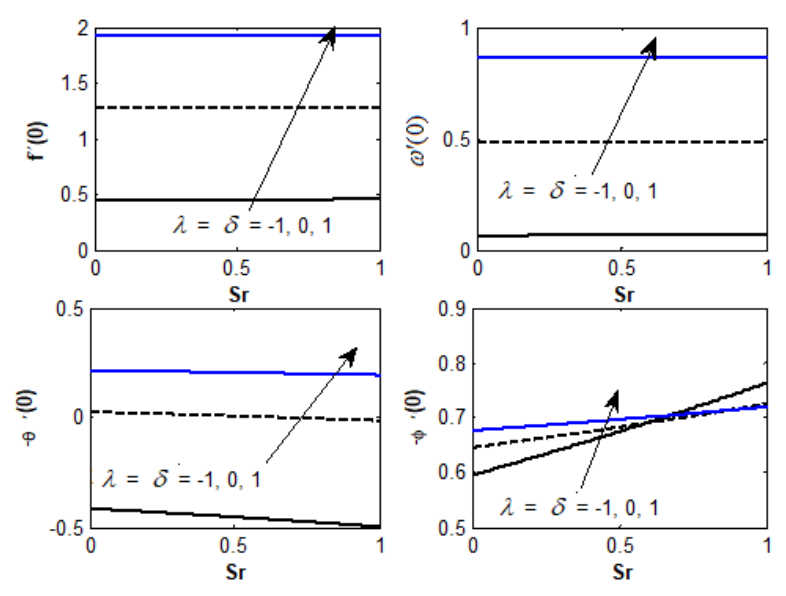

Figure 13. Influence of $\mathrm{Sr}$ on concentration descriptions for $\mathrm{M}=1 ; \mathrm{Kp}=1 ; \mathrm{Pr}=1, \mathrm{Sc}=0.22 ; \mathrm{Du}=0.5 ; \mathrm{S}=-1$
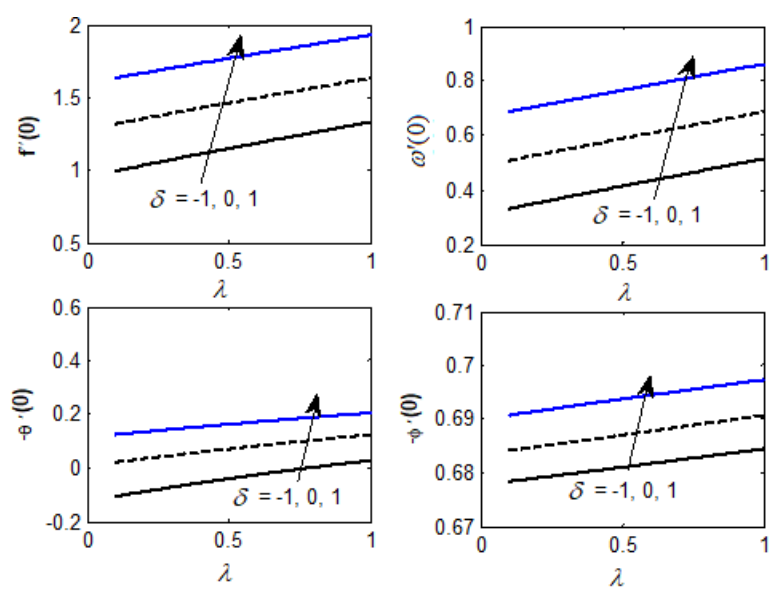

Figure 14. Influence of $\delta$ on profiles for $\mathrm{M}=1 ; \mathrm{Kp}=1$; $\mathrm{Pr}=1$; $\mathrm{Sc}=0.22 ; \mathrm{Sr}=0.5 ; \mathrm{Du}=0.5 ; \mathrm{S}=-1$

\section{CONCLUSION}

A mathematical model has been developed for steady magnetohydrodynamic boundary layer motion of viscous micropolar liquid through a extending surface in a porous medium. The model includes Soret and Dufour effects, MHD, chemical reaction and heat source effects. Numerical solutions are obtained by fourth order Runge-Kutta method. The main findings of the present investigation may be summarized as follows

- The velocity description decreases with an increase in $\Gamma$. For $\Gamma=0$, i.e. in case of Newtonian fluid velocity contributes its maximum value near the plate.

- The non-Newtonian parameter has a decelerating effect for velocity profile.

- The fluid temperature has an enhancing effect by an enhance in material variable.

- Enhancement in Dufour parameter the fluid temperature enhances.

- The skin friction coefficient, couple stress and rate of heat transfer increases with the enhance in thermal buoyancy parameter.

- Thermo-diffusion and Dufour parameters enhance the properties of micropolar liquid. 


\section{ACKNOWLEDGEMENT}

The authors are grateful to Prof G. C. Sharma, Agra University, Agra, India for his help and valuable suggestions to prepare this research paper and thanks to reviewers also

\section{REFERENCES}

[1] Sparrow EM, Cess RD. (1961). The effect of a magnetic field on free convection heat transfer. International Journal of Heat and Mass Transfer 3(4): 267-274. http://doi.org/10.1016/0017-9310(61)90042-4

[2] Gupta AS. (1962). Laminar free convection flow of an electrically conducting fluid from a vertical plate with uniform surface heat flux and variable wall temperature in the presence of a magnetic field. Zeitschrift für angewandte Mathematik und Physik ZAMP 13(4): 324333.

[3] Char MI. (1994). Heat transfer in a hydromagnetic flow over a stretching sheet. Heat Mass Transfer 29(8): $495-$ 500. http://doi.org/10.1007/BF01539502

[4] Chiam TC. (1997). Magnetohydrodynamic heat transfer over a non- isothermal stretching sheet. Acta Mechanica 122(1): 169-179. http://doi.org/10.1007/BF01181997

[5] Liu IC. (2004). Flow and heat transfer of an electrically conducting fluid of second grade over a stretching sheet subject to a transverse magnetic field. International Journal of Heat and Mass Transfer 47(19-20): 4427-4437. http://doi.org/10.1016/j.ijnonlinmec.2004.07.008

[6] Ishak A, Nazar R, Pop I. (2008). Hydromagnetic flow and heat transfer adjacent to a stretching vertical sheet. Heat Mass Transfer 44(8): 921-927. http://doi.org/10.2298/TSCI100308198Y

[7] Prasad KV, Pal D, Datti PS. (2009). MHD power-law fluid flow and heat transfer over a non-isothermal stretching sheet. Communications in Nonlinear Science and Numerical Simulation 14(5): 2178-2189. http://doi.org/10.1016/j.cnsns.2008.06.021

[8] Harris SD, Ingham BD, Pop I. (2009). Mixed convection boundary-layer flow near the stagnation point on a vertical surface in a porous medium: Brinkman model with slip. Transport of Porous Media 77(2): 267-285. http://doi.org/10.1007/s11242-008-9309-6

[9] Aziz A. (2010). Hydrodynamic and thermal slip flow boundary layers over a flat plate with constant heat flux boundary condition. Communications in Nonlinear Science and Numerical Simulation 15(3): 573-580. http://doi.org/10.1016/j.cnsns.2009.04.026

[10] Rohni AM, Ahmad S, Pop I. Merkin JH. (2012). Unsteady mixed convection boundary-layer flow with suction and temperature slip effects near the stagnation point on a vertical permeable surface embedded in a porous medium. Transport of a Porous Media 92(1): 114. http://doi.org/10.1007/s11242-011-9883-x

[11] Tao LN. (1960). On combined free and forced convection in channel. ASME Journal of Heat Transfer 82(3): 233-238. http://doi.org/10.1115/1.3679915

[12] Aung W, Worku G. (1986). Developing flow and flow reversal in a vertical channel with symmetric wall temperatures. ASME Journal of Heat Transfer 108(2) 299-304. http://doi.org/10.1115/1.3246919

[13] Trevisan OV, Bejan A. (1990). Combined heat and mass transfer by natural convection in a porous medium.
Advances in Heat Transfer 20: 315-352.

[14] Barletta A. (1998). Laminar mixed convection with viscous dissipation in a vertical channel. International Journal of Heat and Mass Transfer 41(22): 3501-3513. http://doi.org/10.1016/s0017-9310(98)00074-x

[15] Chamkha AJ, Grosan T, Pop I. (2002). Fully developed free convection of a micropolar fluid in a vertical channel International Communications in Heat and Mass Transfer 29(8): $1119-1127$ http://doi.org/10.1016/S0735-1933(02)00440-2

[16] Ishak A, Nazar R, Pop I. (2008). Magnetohydrodynamic (MHD) flow of a micropolar fluid towards a stagnation point on a vertical surface, 2008, Computers \& Mathematics with Applications 56(12): 3188-3194. http://doi.org/10.1016/j.camwa.2008.09.013

[17] Lok YY, Amin N, Campean D, Pop I. (2005). Steady mixed convection flow of a micropolar fluid near the stagnation point on a vertical surface. International Journal of Numerical Methods for Heat \& Fluid Flow 15(7):

654-670. http://doi.org/10.1108/09615530510613861

[18] Ramachandran N, Chen TS, Armaly BF. (1988). Mixed convection in a stagnation flows adjacent to vertical surfaces. ASME Journal of Heat Transfer 110:2(2): $373-$ 37. http://doi.org/10.1115/1.3250494

[19] Srinivas S, Muthuraj R. (2010). MHD flow with slip effects and temperature-dependent heat source in a vertical wavy porous space. Chemical Engineering Communications 197(11): $1387-1403$ http://doi.org/10.1080/00986441003626102

[20] Srinivas S, Muthuraj R. (2011). Effects of chemical reaction and space porosity on MHD mixed convective flow in a vertical asymmetric channel with peristalsis. Mathematical and Computer Modelling 54(5-6): 12131227.

[21] Nield DA, Bejan A. (1999). Convection in Porous Media. 2nd Edition, Springer, New York. 145-220. http://doi.org/10.1002/0470014164.ch8

[22] Ingham DB, Pop I. (1998). Transport Phenomena in Porous Media. I. Pergamon Oxford.

[23] Ingham DB, Pop I. (2002). Transport Phenomena in Porous Media II, Pergamon, Oxford. http://doi.org/10.1029/EO066i020p00443-03

[24] Bejan A, Khair KR. (1985). Heat and mass transfer by natural convection in a porous medium. International Journal of Heat and Mass Transfer 28: 909-918. http://doi.org/10.1016/0017-9310(85)90272-8

[25] Eckert ERG, Drake RM. (1972). Analysis of Heat and Mass Transfer. McGraw-Hill, New York.

[26] Kafoussias NG, Williams EM. (1995). Thermaldiffusion and diffusion-thermo effects on mixed freeforced convective and mass transfer boundary layer flow with temperature dependent viscosity. International Journal of Engineering Science 33(9): 1369-1383. http://doi.org/10.1016/0020-7225(94)00132-4

[27] Anghel M, Takhar HS, Pop I. (2000). Dufour and Soret effects on free convection boundary-layer over a vertical surface embedded in a porous medium. Studia Universitatis Babes-Bolyai, Mathematica 4: 11-21.

[28] Postelnicu A. (2004). Influence of a magnetic field on heat and mass transfer by natural convection from vertical surfaces in porous media considering Soret and Dufour effects. International Journal of Heat and Mass Transfer 47(6-7):

$1467-1472$ 
http://doi.org/10.1007/s00231-006-0132-8

[29] Postelnicu A. (2007) Influence of chemical reaction on heat and mass transfer by natural convection from vertical surfaces in porous media considering Soret and Dufour effects. Heat Mass Transfer 43(6): 595-602. http://doi.org/10.1007/s00231-006-0132-8

[30] Alam MS, Rahman MM. (2006). Dufour and Soret effects on mixed convection flow past a vertical porous flat plate with variable suction. Nonlinear Analysis: Modelling and Control 11(1): 3-12. http://doi.org/10.1109/HMWC.2014.7000242

[31] Murthy PVSN, Partha MK, Raja Sekhar GP. (2006). Soret and Dufour effects in a non-Darcy porous medium. Journal of Heat Transfer 128(6): 605-610. http://doi.org/10.1115/1.2188512

[32] Rashidi MM, Hayat T, Erfani E, Mohimanian Pour SA, Awatif AH. (2011). Simultaneous effects of partial slip and thermal-diffusion and diffusion-thermo on steady MHD convective flow due to a rotating disk. Communications in Nonlinear Science and Numerical Simulation 16(11): 4303-4317. http://doi.org/10.1016/j.cnsns.2011.03.015

[33] Mishra SR, Dash GC, Acharya M. (2013). Free convective flow of visco-elastic fluid in a vertical channel with Dufour effect. World Applied Sciences Journal 28: 1275-1280.

[34] Baag S, Mishra SR, Dash GC, Acharya MR. (2014). Numerical investigation on MHD microploar fluid flow towards a stagnation point on a vertical surface with heat source and chemical reaction. Journal of King Saud University-Engineering Sciences 3(1). http://doi.org/10.1016/j.jksues.2014.06.002

[35] Bhattacharyya K. (2011). Dual solutions in boundary stagnation-point flow and mass transfer with chemical reaction past a stretching/shrinking sheet. International Communications in Heat and Mass Transfer 38(7): 917 922.

http://doi.org/10.1016/j.icheatmasstransfer.2011.04.020

\section{NOMENCLATURE}

C

D

$\mathrm{Du}$

$\operatorname{Pr}$

Sr

$g$

$M$

$\Gamma$

$\mathrm{Cp}$

\section{Greek symbols}

$T$

$\theta(\eta)$

$T_{\infty}$

$\mu$

Gc

$\delta$

$B_{0}$

$\alpha$

Sh

$\sigma$

$\kappa$

$\lambda$

$N u$

$\rho$

Kc

$v$

Gr

$\eta$

$C_{\infty}$

$\beta_{T}$

$k_{c}^{*}$

$\beta_{c}$

$N$

$\gamma^{\prime}$

$u, v$

$\varphi(\eta)$

j

$k$

$C_{w}$

Ec

$T_{w}$

$x, y$
Permeability parameter

Schmidt number

fluid temperature

dimensionless temperature

fluid temperature at infinity

dynamic viscosity

Grashof number for mass

transfer

solutal buoyancy

parameter

magnetic flux density

thermal diffusivity

Sherwood number

Electrical

thermal conductivity

thermal buoyancy or mixed

convection parameter

Nusselt number

density of the fluid

chemical reaction

parameter

kinematic viscosity

Grashof number for heat

transfer

similarity variable

species concentration at

infinity

thermal expansion

coefficient

reaction rate of the solute

concentration expansion

coefficient

angular Velocity

spin gradient viscosity

velocity components along

$\mathrm{x}$ - and $\mathrm{y}$-direction

non-dimensional

concentration parameter

micro-inertia density

vortex viscosity or

microrotation viscosity

stretching sheet

concentration

Eckert number

stretching sheet

temperature

coordinates 